\title{
The Statistical Properties of the $q$-Deformed Dirac Oscillator in One and Two Dimensions
}

\author{
Abdelmalek Boumali ${ }^{1}$ and Hassan Hassanabadi ${ }^{2}$ \\ ${ }^{1}$ Laboratoire de Physique Appliquée et Théorique, Université Larbi Tébessi, Tébessa, Algeria \\ ${ }^{2}$ Physics Department, Shahrood University of Technology, Shahrood, Iran \\ Correspondence should be addressed to Abdelmalek Boumali; boumali.abdelmalek@gmail.com
}

Received 23 January 2017; Accepted 11 June 2017; Published 16 July 2017

Academic Editor: Edward Sarkisyan-Grinbaum

Copyright ( 2017 Abdelmalek Boumali and Hassan Hassanabadi. This is an open access article distributed under the Creative Commons Attribution License, which permits unrestricted use, distribution, and reproduction in any medium, provided the original work is properly cited. The publication of this article was funded by SCOAP ${ }^{3}$.

\begin{abstract}
We study the behavior of the eigenvalues of the one and two dimensions of $q$-deformed Dirac oscillator. The eigensolutions have been obtained by using a method based on the $q$-deformed creation and annihilation operators in both dimensions. For a twodimensional case, we have used the complex formalism which reduced the problem to a problem of one-dimensional case. The influence of the $q$-numbers on the eigenvalues has been well analyzed. Also, the connection between the $q$-oscillator and a quantum optics is well established. Finally, for very small deformation $\eta$, we (i) showed the existence of well-known $q$-deformed version of Zitterbewegung in relativistic quantum dynamics and (ii) calculated the partition function and all thermal quantities such as the free energy, total energy, entropy, and specific heat. The extension to the case of Graphene has been discussed only in the case of a pure phase $\left(q=e^{i \eta}\right)$.
\end{abstract}

\section{Introduction}

Quantum groups and quantum algebras have attracted much attention of physicists and mathematicians during the last eight years. There had been a great deal of interest in this field, especially after the introduction of the q-deformed harmonic oscillator. Quantum groups and quantum algebras have found unexpected applications in theoretical physics [1]. From the mathematical point of view they are $q$-deformations of the universal enveloping algebras of the corresponding Lie algebras, being also concrete examples of Hopf algebras. When the deformation parameter $q$ is set equal to 1 , the usual Lie algebras are obtained. The realization of the quantum algebra $\mathrm{SU}(2)$ in terms of the $q$-analogue of the quantum harmonic oscillator $[2,3]$ has initiated much work on this topic [4-6]. Biedenharn and Macfarlane [2,3] have studied the $q$-deformed harmonic oscillator based on an algebra of $q$-deformed creation and annihilation operators. They have found the spectrum and eigenvalues of such a harmonic oscillator under the assumption that there is a state with a lowest energy eigenvalue.
Recently, the theory of the q-deformed has become a topic of great interest in the last few years, and it has been finding applications in several branches of physics because of its possible applications in a wide range of areas, such as a $q$-deformation of the harmonic oscillator [7], a $q$-deformed Morse oscillator [8], a classical and quantum $q$-deformed physical systems [9], Jaynes-Cummings model and the deformed-oscillator algebra [10], $q$-deformed supersymmetric quantum mechanics [11], for some modified $q$-deformed potentials [12], on the thermostatistic properties of a $q$ deformed ideal Fermi gas [13], q-deformed Tamm-Dancoff oscillators [14], $q$-deformed fermionic oscillator algebra, and thermodynamics [15], and finally on the fermionic $q$ deformation and its connection to thermal effective mass of a quasiparticle [16].

The relativistic harmonic oscillator is one of the most important quantum systems, as it is one of the very few that can be solved exactly. The Dirac relativistic oscillator (DO) interaction is an important potential both for theory and for application. It was for the first time studied by Itô et al. [17]. They considered a Dirac equation in which the momentum 
$\vec{p}$ is replaced by $\vec{p}-i m \beta \omega \vec{r}$, with $\vec{r}$ being the position vector, $m$ the mass of particle, and $\omega$ the frequency of the oscillator. The interest in the problem was revived by Moshinsky and Szczepaniak [18], who gave it the name of DO because, in the nonrelativistic limit, it becomes a harmonic oscillator with a very strong spin-orbit coupling term. Physically, it can be shown that the DO interaction is a physical system, which can be interpreted as the interaction of the anomalous magnetic moment with a linear electric field $[19,20]$. The electromagnetic potential associated with the $\mathrm{DO}$ has been found by Benitez et al. [21]. The DO has attracted a lot of interests both because it provides one of the examples of the Dirac's equation exact solvability and because of its numerous physical applications [22-27]. Finally, Franco-Villafañe et al. [28] have exposed the proposal of the first experimental microwave realization of the one-dimensional DO.

An important phenomena which we will discuss here is the connection between $(2+1)$ DO with the JaynesCummings JC model, an archetypical quantum optical system: the Jaynes-Cummings model (JCM) is a theoretical model in quantum optics. It describes the system of a twolevel atom interacting with a quantized mode of an optical cavity, with or without the presence of light (in the form of a bath of electromagnetic radiation that can cause spontaneous emission and absorption) [29,30]. The JCM is of great interest in atomic physics, quantum optics, and solid-state quantum information circuits, both experimentally and theoretically. This model describes well the DO interaction and, following Bermudez et al. [31], the exact mapping of the $2+1$ Dirac oscillator onto the Jaynes-Cummings model well established. In this paper, the extension of this connection in the presence of deformation will be our goal.

The q-deformed oscillator systems have attracted much attention and have been considered in many papers (see [32] and references therein). The representation theory of the quantum algebras has led to the development of $q$-deformed oscillator algebra. Since there have been an increasing interest in the study of physical systems using q-oscillator algebra, it has found applications in several branches of physics such as vibrational spectroscopy, nuclear physics, and many body theory and quantum optics. The $q$-analogue of the one-dimensional nonrelativistic harmonic oscillator has been studied by several authors $[2,3,33,34]$. Realizations of the quantum algebra $\mathrm{SU}_{q}(1,1)$ via the one-dimensional $q$ harmonic oscillator were suggested by Chaichian [35]. The representation theory of quantum algebras with a single deformation parameter, $q$, has led to the development of tile now well-known $q$-deformed harmonic oscillator algebra.

In the nonrelativistic case, the investigation of thermodynamic functions of some types of potentials, such as Morse and improved Manning-Rosen potentials and improved Rosen-Morse and Tiez oscillators, through a partition function and its derivatives with respect to temperature, was an important field of research in the literature (see [36-39]). The extension of the nonrelativistic $q$-harmonic oscillator to the relativistic case, to the best of our knowledge, is not available in the literature. In this context and in order the overcome this lack in the literature, the principal aim of this paper will be the study of $q$-deformed DO in one and two dimensions. The concept of $q$-deformation is also applied to the investigation of the connection of $q$-deformed DO with quantum optics and the existence of the wellknown Zitterbewegung in relativistic quantum dynamics of the problem in question. In addition, for small deformation $\eta$, we have evaluated various thermodynamic quantities such as partition function, entropy, and internal energy. Such studies are expected to be relevant when we want to extended them to the case of Graphene. To prove existence of such a system, we want to indicate some papers including applications of $q$-deformations such as study of electronic conductance in disordered metals and doped semiconductors [40], analyzing of the phonon spectrum in ${ }^{4} \mathrm{He}$ [41], and expression of the oscillatory-rotational spectra of diatomic and multiatomic molecules [42-46].

The structure of this paper is as follows: Section 2 is devoted to the case of the standard $q$-harmonic oscillator. The extension to the $q$-deformed DO will be treated in Section 3 . Different numerical results about the thermal properties of $q$-deformed DO are discussed in Section 4. Finally, Section 5 will be the conclusions.

\section{2. $q$-Deformed One-Dimensional Harmonic Oscillator}

The Hamiltonian of the $q$-deformed harmonic oscillator is

$$
H=\frac{P_{q}^{2}}{2 m}+\frac{1}{2} m \omega^{2} Q_{q}^{2},
$$

where the $q$-momentum $\left(P_{q}\right)$ and $q$-position $\left(Q_{q}\right)$ operators are directly written in terms of the $q$-boson operators $a$ and $a^{+}$as

$$
\begin{aligned}
& P_{q}=i \sqrt{\frac{m \omega \hbar}{2}}\left(a-a^{+}\right), \\
& Q_{q}=\sqrt{\frac{\hbar \omega}{2 m}}\left(a+a^{+}\right),
\end{aligned}
$$

with

$$
\begin{gathered}
{\left[a, a^{+}\right]_{q}=a a^{+}-q^{-1} a^{+} a=q^{N},} \\
a|0\rangle=0,|n\rangle=\frac{\left(a^{+}\right)^{n}}{\sqrt{[n] !}}|0\rangle .
\end{gathered}
$$

Both $q$-factorial and $q$-numbers are defined, respectively, by

$$
\begin{aligned}
{[n] ! } & =[n][n-1] \cdots[1], \\
{[n] } & =\frac{q^{n}-q^{-n}}{q-q^{-1}} .
\end{aligned}
$$

Here $N=a^{+} a$ is the number operator. The eigenvalues of the $q$-deformed one-dimensional harmonic oscillator are [47]

$$
E_{n}=\frac{\hbar \omega}{2}([n]+[n+1]) .
$$


Following (5) and (6), two cases can be distinguished.

(i) When we put $q=e^{\eta}$, the eigenvalues become

$$
E_{n}=\frac{\hbar \omega}{2} \frac{\sinh [\eta(n+1 / 2)]}{\sinh (\eta / 2)} .
$$

(ii) Now, when we write $q=e^{i \eta}$, the form of the spectrum, in this case, is

$$
E_{n}=\frac{\hbar \omega}{2} \frac{\sin [\eta(n+1 / 2)]}{\sin (\eta / 2)} .
$$

In both cases, when $q \rightarrow 1(\eta \rightarrow 0)$ the well-known relation

$$
E_{n}=\hbar \omega\left(n+\frac{1}{2}\right)
$$

is recovered.

Thus, according to (7) and (8), one can see that for $q$ real $\left(q=e^{\eta}\right)$ the energy eigenvalues increase more rapidly than the ordinary case, and the spectrum, in this case, gets expanded. In contrast, when $q$ is a pure phase $\left(q=e^{i \eta}\right)$, the eigenvalues of the energy increase less rapidly than the ordinary case; that is, the spectrum is squeezed [47].

In what follows, we treat the case of the one- and twodimensional $q$-deformed DO.

\section{Solutions of a $q$-Deformed Dirac Oscillator}

3.1. One-Dimensional q-Deformed Dirac Oscillator. The onedimensional DO is

$$
\left\{c \alpha_{x}\left(P_{q}-i m \omega \beta Q_{q}\right)+\beta m c^{2}\right\} \psi_{D}=\epsilon \psi_{D},
$$

with $\psi_{D}=\left(\begin{array}{ll}\psi_{1} & \psi_{2}\end{array}\right)^{T}, \alpha_{x}=\sigma_{x}$, and $\beta=\sigma_{z}$. In this case, (10) becomes

$$
H_{D} \psi_{D}=\epsilon \psi_{D}
$$

with

$$
H_{D}=\left(\begin{array}{cc}
m c^{2} & c\left(p_{x}+i m \omega x\right) \\
c\left(p_{x}-i m \omega x\right) & -m c^{2}
\end{array}\right)
$$

by introducing the usual annihilation and creation operators of the $q$-deformed harmonic oscillator

$$
\begin{aligned}
& P_{q}=i \sqrt{\frac{m \omega \hbar}{2}}\left(a-a^{+}\right), \\
& Q_{q}=\sqrt{\frac{\hbar \omega}{2 m}}\left(a+a^{+}\right),
\end{aligned}
$$

this Hamiltonian transforms into

$$
H_{D}=\left(\begin{array}{cc}
m c^{2} & g a^{\dagger} \\
g^{*} a & -m c^{2}
\end{array}\right)
$$

with $g=i m c^{2} \sqrt{2 r}$ being the coupling strength between orbital and spin degrees of freedom, and $r=\hbar \omega / m c^{2}=$ 1 is a parameter which controls the nonrelativistic limit. It is an important parameter that specifies the importance of relativistic effects in the DO.

Writing that $\psi_{D}=(|n\rangle,|n-1\rangle)^{T}$, this equation can be solved algebraically. Following the above section, when $q$ is real, the spectrum of energy is

$$
\epsilon_{n}= \pm m c^{2} \sqrt{1+2 \frac{\sinh (\eta n)}{\sinh (\eta)}}
$$

Now, if $q$ is complex, it becomes

$$
\bar{\epsilon}_{n}= \pm m c^{2} \sqrt{1+2 \frac{\sin (\eta n)}{\sin (\eta)}} .
$$

In both cases, when $q \rightarrow 1(\eta \rightarrow 0)$ the well-known relation

$$
\widetilde{\epsilon}_{n}= \pm m c^{2} \sqrt{1+2 n}
$$

is recovered [25]. The eigensolutions of a two-dimensional $\mathrm{DO}$, in both cases, can be written as

$$
|\psi\rangle=\left[\begin{array}{c}
\sqrt{\frac{E_{n} \pm m c^{2}}{2 E_{n l}}}|n\rangle \\
\mp i \sqrt{\frac{E_{n} \mp m c^{2}}{2 E_{n l}}}|n-1\rangle
\end{array}\right] .
$$

Using the creation and annihilation operators and the raising and lowering operators $\sigma^{ \pm}=(1 / 2)\left(\sigma^{x} \pm i \sigma^{y}\right)$ Dirac Spinor one can rewrite the previous equation as

$$
H_{1 D}=g\left(\sigma^{\dagger} a+\sigma^{-} a^{\dagger}\right)+\Delta \sigma_{z}
$$

This Hamiltonian is exactly the JCM Hamiltonian in quantum optics [48]. Thus the one-dimensional DO maps exactly onto the Jaynes-Cummings (JC), provided that one identifies the isospin with the atomic system and the spatial degrees of freedom with the cavity mode. Thus, as a result, the relativistic Hamiltonian of a $q$-deformed one-dimensional DO can be mapped onto a $q$-deformed Jaynes-Cummings (JC).

\subsection{Two-Dimensional q-Deformed Dirac Oscillator}

3.2.1. Complex Formalism. In terms of complex coordinates and its complex conjugate, we have

$$
\begin{aligned}
z & =x+i y, \\
\bar{z} & =x-i y, \\
\frac{\partial}{\partial z} & =\frac{1}{2}\left(\frac{\partial}{\partial x}-i \frac{\partial}{\partial y}\right), \\
\frac{\partial}{\partial \bar{z}} & =\frac{1}{2}\left(\frac{\partial}{\partial x}+i \frac{\partial}{\partial y}\right) .
\end{aligned}
$$


The operators momenta $p_{x}$ and $p_{y}$, in the Cartesian coordinates, are defined by

$$
\begin{aligned}
& p_{x}=-i \hbar \frac{\partial}{\partial x} \\
& p_{y}=-i \hbar \frac{\partial}{\partial y} .
\end{aligned}
$$

When we use $p_{z}=-i \hbar(\partial / \partial z)$, we get

$$
\begin{aligned}
& p_{z}=-i \hbar \frac{d}{d z}=\frac{1}{2}\left(p_{x}-i p_{y}\right), \\
& \bar{p}_{z}=-i \hbar \frac{d}{d \bar{z}}=\frac{1}{2}\left(p_{x}+i p_{y}\right),
\end{aligned}
$$

with $p_{z}=-\bar{p}_{z}$. These operators obey the basic commutation relations

$$
\begin{aligned}
& {\left[z, p_{z}\right]=\left[\bar{z}, p_{\bar{z}}\right]=i \hbar} \\
& {\left[z, p_{\bar{z}}\right]=\left[\bar{z}, p_{z}\right]=0 .}
\end{aligned}
$$

The usual creation and annihilation operators, $a_{x}$ and $a_{y}$, with

$$
\begin{aligned}
& a_{x}=\sqrt{\frac{m \omega}{2 \hbar}} x+i \frac{1}{\sqrt{2 m \omega \hbar}} p_{x}, \\
& a_{y}=\sqrt{\frac{m \omega}{2 \hbar}} y+i \frac{1}{\sqrt{2 m \omega \hbar}} p_{y}
\end{aligned}
$$

can be reformulated, in the formalism complex, as follows:

$$
\begin{aligned}
& a_{z}=i\left(\frac{1}{\sqrt{m \omega \hbar}} \bar{p}_{z}-\frac{i}{2} \sqrt{\frac{m \omega}{\hbar}} z\right), \\
& \bar{a}_{z}=-i\left(\frac{1}{\sqrt{m \omega \hbar}} p_{z}+\frac{i}{2} \sqrt{\frac{m \omega}{\hbar}} \bar{z}\right) .
\end{aligned}
$$

These operators, also, satisfy the habitual commutation relations

$$
\begin{aligned}
& {\left[a_{z}, \bar{a}_{z}\right]=1,} \\
& {\left[a_{z}, a_{z}\right]=0,} \\
& {\left[\bar{a}_{z}, \bar{a}_{z}\right]=0 .}
\end{aligned}
$$

Now, In the case of $q$-deformed DO, the creation and annihilation operators $\bar{a}_{z}$ and $a_{z}$ satisfy the commutation relation

$$
\left[a_{z}, \bar{a}_{z}\right]_{q}=a_{z} \bar{a}_{z}-q^{-1} \bar{a}_{z} a_{z}=q^{N}
$$

where $N$ is the number operator, satisfying

$$
\begin{aligned}
& {\left[N, \bar{a}_{z}\right]=\bar{a}_{z},} \\
& {\left[N, a_{z}\right]=-a_{z} .}
\end{aligned}
$$

3.2.2. The Solutions. The two-dimensional DO is

$$
\left[c \sigma_{x}\left(p_{x}-i m \omega x\right)+c \sigma_{y}\left(p_{y}-i m \omega y\right)\right] \psi=\varepsilon \psi,
$$

with $\psi_{D}=\left(\begin{array}{ll}\psi_{1} & \psi_{2}\end{array}\right)^{T}, \alpha_{x}=\sigma_{x}$, and $\beta=\sigma_{z}$. With definitions of Dirac matrices,

$$
\begin{aligned}
& \alpha_{x}=\sigma_{x}=\left(\begin{array}{ll}
0 & 1 \\
1 & 0
\end{array}\right), \\
& \alpha_{y}=\sigma_{y}=\left(\begin{array}{cc}
0 & -i \\
i & 0
\end{array}\right),
\end{aligned}
$$

(8) can be decoupled in a set of equations as follows:

$$
\begin{aligned}
& \varepsilon\left|\psi_{1}\right\rangle=c\left(p_{x}+i m \omega x-i p_{y}+m \omega y\right)\left|\psi_{2}\right\rangle, \\
& \varepsilon\left|\psi_{2}\right\rangle=c\left(p_{x}-i m \omega x+i p_{y}+m \omega y\right)\left|\psi_{1}\right\rangle,
\end{aligned}
$$

and so (9) reads

$$
\begin{aligned}
& H_{D} \\
& =\left(\begin{array}{cc}
m c^{2} & c\left(p_{x}+i m \omega x-i p_{y}+m \omega y\right) \\
c\left(p_{x}-i m \omega x+i p_{y}+m \omega y\right) & -m c^{2}
\end{array}\right) .
\end{aligned}
$$

This last form of Hamiltonian of Dirac can be written, in the complex formalism, by

$$
\begin{aligned}
H_{D} & =\left(\begin{array}{cc}
m c^{2} & 2 c p_{z}+i m \omega c \bar{z} \\
2 c \bar{p}_{z}-i m \omega c z & -m c^{2}
\end{array}\right) \\
& =\left(\begin{array}{cc}
m c^{2} & 2 g \bar{a}_{z} \\
2 g^{*} a_{z} & -m c^{2}
\end{array}\right) .
\end{aligned}
$$

Thus the problem is transformed to the one-dimensional case with a complex variable $z$.

Now, following (9) and (10), the wave functions $\psi_{1}$ and $\psi_{2}$ can be rewritten in the language of the complex annihilationcreation operators as

$$
\begin{aligned}
& \left|\psi_{1}\right\rangle=\frac{g}{\varepsilon-m c^{2}} \bar{a}_{z}\left|\psi_{2}\right\rangle, \\
& \left|\psi_{2}\right\rangle=\frac{g^{*}}{\varepsilon+m c^{2}} a_{z}\left|\psi_{1}\right\rangle .
\end{aligned}
$$

When we write the component $\left|\psi_{1}\right\rangle$ in terms of the quanta bases, $|n\rangle=\left(\left(a^{\dagger}\right)^{n} / \sqrt{[n] !}\right)|0\rangle$, these equations can be simultaneously diagonalized, and the energy spectrum can be described by

$$
\varepsilon_{n}= \pm m c^{2} \sqrt{1+4 \frac{\sinh [\eta n]}{\sinh (\eta)}}
$$

when $q$ is real and by

$$
\bar{\varepsilon}_{n}= \pm m c^{2} \sqrt{1+4 \frac{\sin [\eta n]}{\sin (\eta)}}
$$


if $q$ is complex. In both cases, when $q \rightarrow 1(\eta \rightarrow 0)$ the wellknown relation

$$
\widetilde{\varepsilon}_{n}= \pm m c^{2} \sqrt{1+4 n}
$$

is recovered. Our results are in good agreement with those obtained by Hatami and Setare [49].

In order to establish the connection between a twodimensional $q$-deformed DO and quantum optics, the Dirac Hamiltonian can be rewritten into another form as

$$
H_{2 D}=g\left(\sigma^{\dagger} \bar{a}_{z}+\sigma^{-} a_{z}\right)+\Delta \sigma_{z}
$$

This form of the Hamiltonian corresponds to the $q$-deformed Anti-Jaynes-Cummings (AJC) model. Here $\sigma^{ \pm}=(1 / 2)\left(\sigma^{x} \pm\right.$ $\left.i \sigma^{y}\right)$ are the spin arising and lowering operators, and $\Delta=$ $m c^{2}$ is a detuning parameter. As a result, the relativistic Hamiltonian of a $q$-deformed two-dimensional DO can be mapped onto a couple of $q$-deformed Anti-Jayne-Cummings (AJC) which describes the interaction between the relativistic spin and bosons.

We further observe that the Zitterbewegung frequency for the $q$-deformed $(2+1)$-dimensional DO depends on the parameter of deformation $\eta$. To show this we, first, start with the following eigensolutions of a two-dimensional DO:

$$
\left|\psi_{1,2}\right\rangle=\left[\begin{array}{c}
\sqrt{\frac{E_{n} \pm m c^{2}}{2 E_{n l}}}|n\rangle \\
\mp i \sqrt{\frac{E_{n} \mp m c^{2}}{2 E_{n l}}}|n-1\rangle
\end{array}\right] .
$$

Here, $E_{n} \equiv \epsilon_{n}$ (or $E_{n} \equiv \varepsilon_{n}$ ) for $q$ real (or $q$ complex), respectively. The eigenstates can be expressed transparently in terms of two-component Pauli spinors $\left|\chi_{\uparrow}\right\rangle$ and $\left|\chi_{\downarrow}\right\rangle$ [31]:

$$
\begin{aligned}
& \left|\psi_{1}\right\rangle=\alpha_{n}|n\rangle\left|\chi_{\uparrow}\right\rangle-i \gamma_{n}|n-1\rangle\left|\chi_{\downarrow}\right\rangle, \\
& \left|\psi_{2}\right\rangle=\gamma_{n}|n\rangle\left|\chi_{\uparrow}\right\rangle+i \alpha_{n}|n-1\rangle\left|\chi_{\downarrow}\right\rangle,
\end{aligned}
$$

where $\alpha_{n}=\sqrt{\left(\varepsilon_{n}+m c^{2}\right) / 2 \epsilon_{n}}$ (or $\left.\sqrt{\left(\bar{\varepsilon}_{n}+m c^{2}\right) / 2 \varepsilon_{n}}\right)$ and $\delta_{n}=\sqrt{\left(\varepsilon_{n}-m c^{2}\right) / 2 \epsilon_{n}}\left(\right.$ or $\sqrt{\left.\left(\bar{\varepsilon}_{n}-m c^{2}\right) / 2 \varepsilon_{n}\right)}$ are real. Following these equations, the eigenstates present entanglement between the orbital and spin degrees of freedom. To clarify this, we start with some initial pure state at $t=0$,

$$
|\Psi(0)\rangle=|n-1\rangle\left|\chi_{\uparrow}\right\rangle=i \alpha_{n}\left|\psi_{1}\right\rangle-i \gamma_{n}\left|\psi_{2}\right\rangle .
$$

This equation shows that the starting initial state is a superposition of both the positive and negative energy solutions, which is the fundamental ingredient that leads to Zitterbewegung in relativistic quantum dynamics.

The evolution of this initial state can be expressed as

$$
|\Psi(t)\rangle=i \gamma_{n} e^{-i \omega_{n} t}\left|\psi_{1}\right\rangle-i \alpha_{n} e^{i \omega_{n} t}\left|\psi_{2}\right\rangle,
$$

where

$$
\omega_{n}=\frac{\epsilon_{n}}{\hbar}=\frac{m c^{2}}{\hbar} \sqrt{1+4 \frac{\sinh (\eta n)}{\sinh (\eta)}}
$$

for $q$ real and

$$
\omega_{n}=\frac{\bar{\epsilon}_{n}}{\hbar}=\frac{m c^{2}}{\hbar} \sqrt{1+4 \frac{\sin (\eta n)}{\sin (\eta)}}
$$

for $q$ complex. In both cases, $\omega_{n}$ describes the frequency of oscillations: the frequency oscillation between positive and negative energy solutions.

If we consider very small deformation and neglect all terms proportional to $\eta^{4}$, we have

$$
\begin{aligned}
\omega_{n} & =\frac{\epsilon_{n}}{\hbar}=\frac{m c^{2}}{\hbar} \sqrt{1+4 \frac{\sinh [\eta n]}{\sinh (\eta)}} \\
& \approx \frac{m c^{2}}{\hbar} \sqrt{1+4 n+\frac{2}{3} \eta^{2} n^{3}} \\
& \approx \omega_{D O}\left(1 \pm \frac{1}{3} \frac{n^{3}}{(1+4 n)} \eta^{2}\right),
\end{aligned}
$$

with $\omega_{D O}=\left(m c^{2} / \hbar\right) \sqrt{1+4 n}$ being the frequency of a twodimensional DO without deformation, and the sign + denotes the case for $q$ real and the sign - denotes the case of $q$ complex. In the approximation of very small $\eta$, the final form will be

$$
|\Psi(t)\rangle=\underbrace{|\Psi(t)\rangle_{\eta=0}}_{\text {without deformation }}+\eta^{2} \underbrace{|\Psi(t)\rangle_{\eta \neq 0}}_{\text {with deformation }},
$$

where

$$
\begin{aligned}
& |\Psi(t)\rangle_{\eta=0}=\left(\cos \omega_{D O} t+\frac{i \sin \omega_{D O} t}{\sqrt{1+4 n}}\right)\left|\psi_{1}\right\rangle \\
& +\sqrt{\frac{4 n}{1+4 n}} \sin \omega_{D O} t\left|\psi_{2}\right\rangle, \\
& |\Psi(t)\rangle_{\eta \neq 0}=\left(\left[\frac{1}{3} \frac{n^{3} \omega_{D O} t}{(1+4 n)} \sin \omega_{D O} t+\frac{i}{\sqrt{1+4 n}}\left\{\mp \frac{1}{3}\right.\right.\right. \\
& \left.\left.\left.+\frac{n^{3} \omega_{D O} t}{(1+4 n)}\left(\cos \omega_{D O} t+\sin \omega_{D O} t\right)\right\}\right]\right)\left|\psi_{1}\right\rangle \\
& +\left\{\sqrt{\frac{4 n}{1+4 n}} \frac{1}{3} \frac{n^{3} \omega_{D O} t}{(1+4 n)} \cos \omega_{D O} t-\sqrt{\frac{4 n}{1+4 n}}\left(\frac{n^{2}}{12}\right.\right. \\
& \left.\left.+\frac{1}{3} \frac{n^{3}}{(1+4 n)}\right) \sin \omega_{D O} t\right\}\left|\psi_{2}\right\rangle .
\end{aligned}
$$

The sign (-) denotes the case of $q$ real and $(+)$ denotes the other case (pure phase). This equation shows the oscillatory behavior between the states $|n\rangle\left|\chi_{\uparrow}\right\rangle$ and $|n-1\rangle\left|\chi_{\downarrow}\right\rangle$ which is exactly similar to atomic Rabi oscillations occurring in the JC and AJC models. The $q$-deformed Rabi frequency, $\omega_{n}$, follows (46) for both cases.

3.3. Discussions. This section is devoted to study the influence of $q$-deformed algebra on the eigenvalues of the DO in one and two dimensions. This influence has been well 

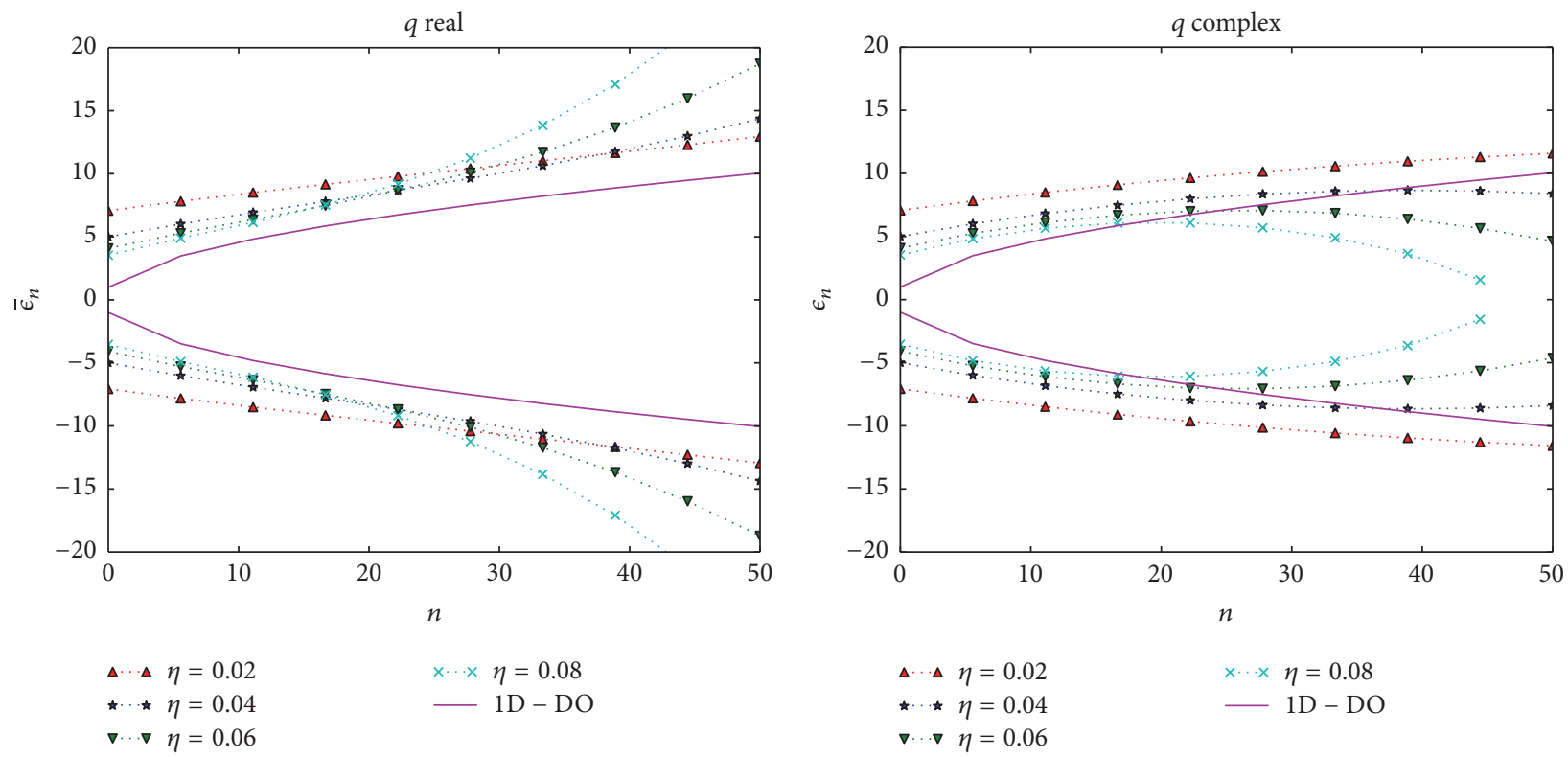

(a) $q$-deformed one-dimensional Dirac oscillator
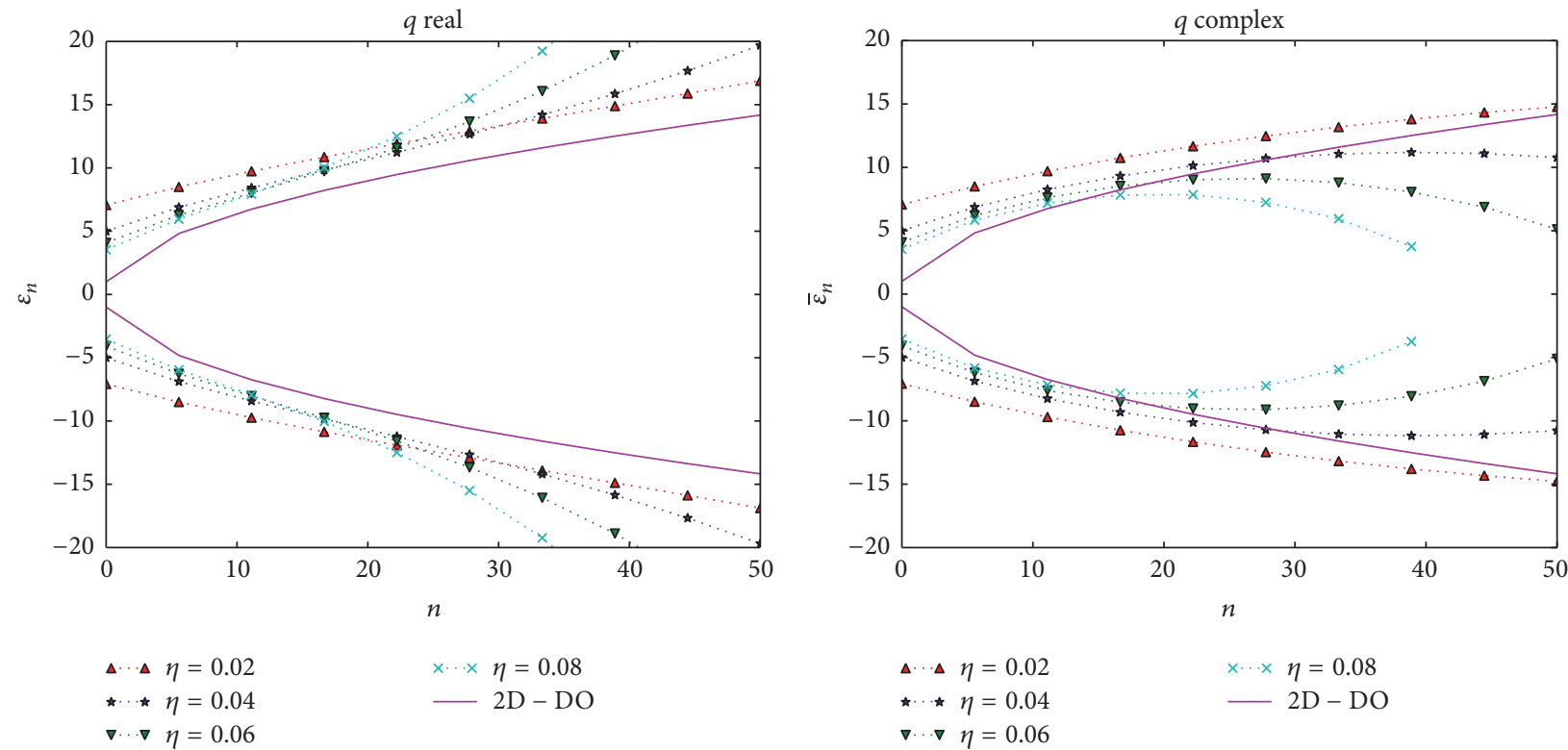

(b) $q$-deformed two-dimensional Dirac oscillator

Figure 1: Spectrum of energy of one- and two-dimensional Dirac oscillator versus quantum number for both cases of $q$.

established through the parameter $\eta$ with $q=e^{\eta}$. In Figure 1 , we present the eigenvalues of the $q$-deformed DO in one and two dimensions versus the quantum number with different values of parameter $\eta$ in both cases of $q$ real and complex. In order to discuss the results, we use the same explication used by Neskovic and Urosevic [50] in their study of the statistical properties of quantum oscillator: thus, the energy levels of the $q$-oscillator are not uniformly spaced for $q=1$. The behavior of the energy spectra is completely different in the cases $q=e^{i \eta}$ and $q=e^{\eta}$. When $q$ is real $q=e^{i \eta}$, the separation between the levels increases with the value of $n$; that is, the spectrum is extended. On the other hand, when $q$ is a pure phase, the separation between all the levels decreases with increasing $n$; that is, the spectrum is squeezed.

In Figure 2 we present the frequency $\omega_{n}$ versus a quantum number $n$ for both $q$ real and complex in one and two dimensions: this frequency describes the oscillations between positive and negative energy solutions. As a consequence, we are in the case of well-known Zitterbewegung in relativistic quantum dynamics. This phenomenon, due to the interference of positive and negative energies, has never been observed experimentally. The reason is that the amplitude of 

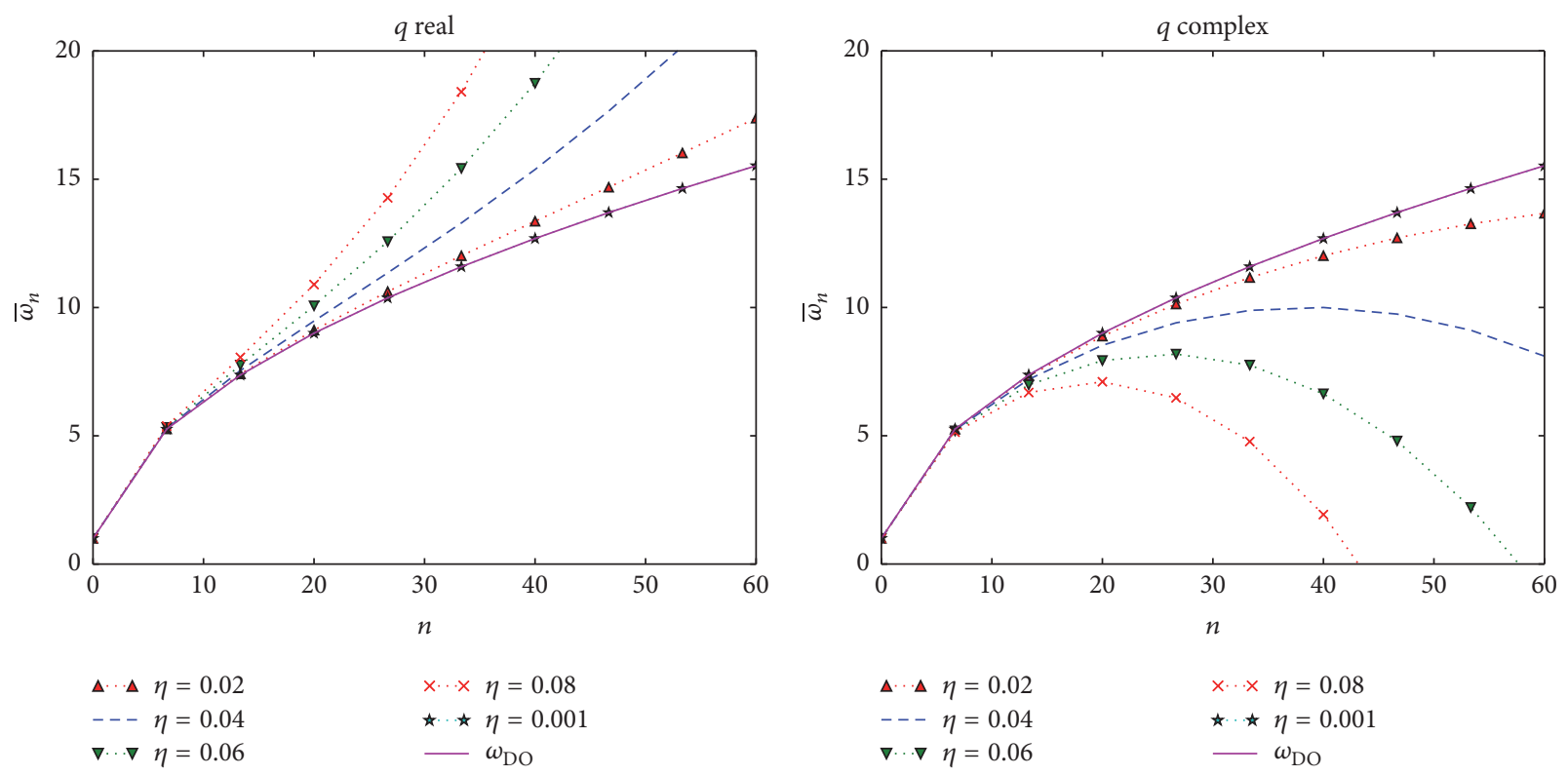

FIGURE 2: Reduced frequency versus quantum number: here $\hbar=m=c=1$.

these rapid oscillations lies below the Compton wavelength. The influence of the deformation on this frequency is well established also.

Finally, the exact connection of the $q$-deformed DO with both Jaynes-Cummings (JC) and anti-Jaynes-Cummings (AJC) models has been established.

\section{Thermal Properties of $q$-Deformed Dirac Oscillator}

The probability of finding a system in a state with energy $E_{n}$ is given by $[50,51]$

$$
P_{n}=\frac{e^{-E_{n} / k_{B} T}}{Z},
$$

where $Z=\operatorname{tr} e^{-H / k_{B} T}=\sum e^{-\beta E_{n}}$ is the partition function. In what follows, our main object is to obtain this partition function for small deformation $\eta$ : in this case the summation appearing in $Z$ can be easily performed, and all thermodynamic potentials such as free energy $F$, entropy $S$, total energy $U$, and specific heat can be calculated. Before doing so, lets rewrite the form of energy in a more convenient form, starting with the following equation:

$$
\epsilon_{n}= \pm m c^{2} \sqrt{1+a \frac{\sinh (\eta n)}{\sinh (\eta)}}
$$

for $q$ real and

$$
\bar{\epsilon}_{n}= \pm m c^{2} \sqrt{1+a \frac{\sin (\eta n)}{\sin (\eta)}}
$$

for $q$ complex. Here $a=2$ for one-dimensional case (and $a=$ 4 for a two-dimensional case). Now, in order to extract these properties of our q-oscillator, we will only restrict ourselves to stationary states of positive energy. The DO possesses an exact Foldy-Wouthuysen transformation (FWT): so, the positiveand negative-energy solutions never mix. Following this, we only consider the positive part of energy.

As $\sinh (\eta n) / \sinh (\eta)$ is even as a function of $\eta$, the same property has the energy and all quantities derived from it [50]. Now, we will consider very small deformation and neglect all terms proportional to $\eta^{4}$. In this case, we have

$$
\epsilon_{n} \simeq \sqrt{1+a n}\left(1+\frac{a n^{3}}{12(1+a n)} \eta^{2}\right) .
$$

With the same argument, the energy spectrum of the $q$ complex case can be written as

$$
\bar{\epsilon} \simeq \sqrt{1+a n}\left(1-\frac{a n^{3}}{12(1+a n)} \eta^{2}\right) .
$$

A both equations can be written in a compact form as

$$
\xi_{n}=\sqrt{1+a n}\left(1 \pm \frac{a n^{3}}{12(1+a n)} \eta^{2}\right)
$$

or

$$
\xi_{n}=\xi_{n 0}+\xi_{n \pm},
$$

with

$$
\xi_{n 0}=\sqrt{1+a n}
$$

being the reduced spectrum of energy of the ordinary DO, and

$$
\xi_{n \pm}= \pm \frac{a n^{3}}{12 \sqrt{1+a n}} \eta^{2}
$$

is the correction on the energy when the deformation exists. 
In this case, the partition function is

$$
\begin{aligned}
Z & =\sum_{n=0}^{\infty} e^{-\xi_{n \pm} / \tau} \simeq \sum_{n=0}^{\infty} e^{-\sqrt{1+a n} / \tau}\left(1 \pm \frac{a n^{3}}{12 \tau \sqrt{1+a n}} \eta^{2}\right) \\
& =Z_{0}+Z_{1}
\end{aligned}
$$

with $\tau=k_{B} T / m c^{2}$ and

$$
\begin{aligned}
& Z_{0}=\sum_{n=0}^{\infty} e^{-\sqrt{1+a n} / \tau}, \\
& Z_{1}= \pm \frac{a \eta^{2}}{12 \tau} \sum_{n=0}^{\infty} \frac{n^{3}}{\sqrt{1+a n}} e^{-\sqrt{1+a n} / \tau} .
\end{aligned}
$$

In order to evaluate the first term, $Z_{0}$, we use a method based on Zeta function: so, by using the formula $[25,26]$

$$
e^{-x}=\frac{1}{2 \pi i} \int_{C} d s x^{-s} \Gamma(s),
$$

the sum in (12) is transformed into

$$
\begin{aligned}
\sum_{n} e^{-(\gamma / \tau) \sqrt{\alpha+n}} & =\frac{1}{2 \pi i} \int_{C} d s\left(\frac{\gamma}{\tau}\right)^{-s} \sum_{n}(n+\alpha)^{-s / 2} \Gamma(s) \\
& =\frac{1}{2 \pi i} \int_{C} d s\left(\frac{\gamma}{\tau}\right)^{-s} \zeta_{H}\left(\frac{s}{2}, \alpha\right) \Gamma(s),
\end{aligned}
$$

with $x=(\gamma / \tau) \sqrt{\alpha+n}, \gamma=\sqrt{a}, \alpha=1 / a$. Here, $\Gamma(s)$ and $\zeta_{H}(s / 2, \alpha)$ are, respectively, the Euler and Hurwitz zeta functions. Applying the residues theorem, for the two poles $s=0$ and $s=2$, the desired partition function is written down in terms of the Hurwitz zeta function as follows:

$$
Z_{0}(\tau)=\frac{\tau^{2}}{a}+\zeta_{H}\left(0, \frac{1}{a}\right) .
$$

Now, the second term can be evaluated by using the EulerMaclaurin formula; starting by the equation

$$
Z_{1}= \pm \frac{a \eta^{2}}{12 \tau} \sum_{n=0}^{\infty} \frac{n^{3}}{\sqrt{1+a n}} e^{-\sqrt{1+a n} / \tau}
$$

and, according to this approach, the sum transforms to the integral as follows:

$$
\begin{aligned}
\sum_{x=0}^{\infty} f(x)= & \frac{1}{2} f(0)+\int_{0}^{\infty} f(x) d x \\
& -\sum_{p=1}^{\infty} \frac{B_{2 p}}{(2 p) !} f^{(2 p-1)}(0) .
\end{aligned}
$$

Here $f(x)=\left(x^{3} / \sqrt{1+a x}\right) e^{-\sqrt{1+a x} / \tau}, B_{2 p}$ are the Bernoulli numbers, and $f^{(2 p-1)}$ is the derivative of order $(2 p-1)$. Up to $p=1$, the final form of $Z_{1}$ term is

$$
\begin{aligned}
Z_{I}(\tau, \eta) & =\int_{0}^{\infty} f(x) d x \\
& =b \eta^{2} e^{-1 / \tau}\left(15 \tau^{6}+15 \tau^{5}+6 \tau^{4}+\tau^{3}\right),
\end{aligned}
$$

with $b=6 e^{-1 / \tau}$ when $a=2$, and $b=(3 / 8) e^{-1 / \tau}$ when $a=4$.
Finally, the compact final form of the $q$-deformed partition function of the DO in one and two dimensions for both cases of $q$ is

$$
\begin{aligned}
Z_{q}(\tau, \eta)= & \frac{\tau^{2}}{2}+\zeta_{H}\left(0, \frac{1}{a}\right) \\
& \pm \eta^{2} b\left(15 \tau^{6}+15 \tau^{5}+6 \tau^{4}+\tau^{3}\right) .
\end{aligned}
$$

Here, the sign (-) describes the partition function in the case where $q$ is real and $(+)$ describes the case of $q$ complex.

Via (30), the determination of all thermal properties, such as the free energy, the entropy, total energy, and the specific heat, can be obtained through the numerical partition function $Z(\tau)$ by using the following relations [26]:

$$
\begin{aligned}
F & =-\tau \ln (Z) \\
U & =\tau^{2} \frac{\partial \ln (Z)}{\partial \tau} \\
\frac{S}{k_{B}} & =\ln (Z)+\tau \frac{\partial \ln (Z)}{\partial \tau} \\
\frac{C}{k_{B}} & =2 \tau \frac{\partial \ln (Z)}{\partial \tau}+\tau^{2} \frac{\partial^{2} \ln (Z)}{\partial \tau^{2}} .
\end{aligned}
$$

4.1. Numerical Results. Figure 3 shows the thermal properties of a one- and two-dimensional $q$-deformed DO in both cases of $q$. from this figure, we can confirm that the deformation plays a significant role in these properties, and the effect of the parameters is very important on the thermodynamic properties. Also, the behavior of these quantities is completely different in the cases $q=e^{i \eta}$ and $q=e^{\eta}$. When $q$ is a pure phase, the behavior of different thermal quantities has a similar comportment as in the case of nondeformed DO in both one and two dimensions $[25,26]$. On the other hand if $q$ is real, these quantities show a strange behavior. This situation is closely related to the nature of spectrum: if $q$ is real, the spectrum is extended which is the cause of the strange behavior of the thermodynamics quantities, contrary to the case where $q$ is complex where the spectrum is squeezed: in this case we obtain the same form of all curves in these quantities. In what follows, in order to compare our results with those obtained in literature, we focus on the case of pure phase.

We should mention that, in all figures, we have used dimensionless quantities, and the temperature range is taken from $10^{8} \mathrm{~K}$ to $10^{14} \mathrm{~K}$. These values give an order of the oscillator frequency about $10^{20} \mathrm{~Hz}$ similar to that of Zitterbewegung frequency in the DO, which has so far been experimentally inaccessible. For the asymptotic limits, as are shown by the figures of the specific heat in the presence of deformation, all curves coincide and reach the fixed value $C=6 k_{B}$ three times greater compared to the case of nondeformed DO in one and two dimensions.

As an application, we can extend our calculations to the case of Graphene: Graphene is a two-dimensional configuration of carbon atoms organized in a hexagonal honeycomb 


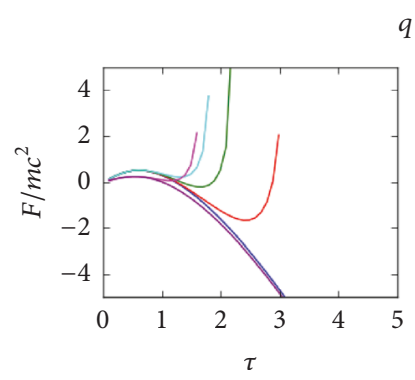

$q$ real
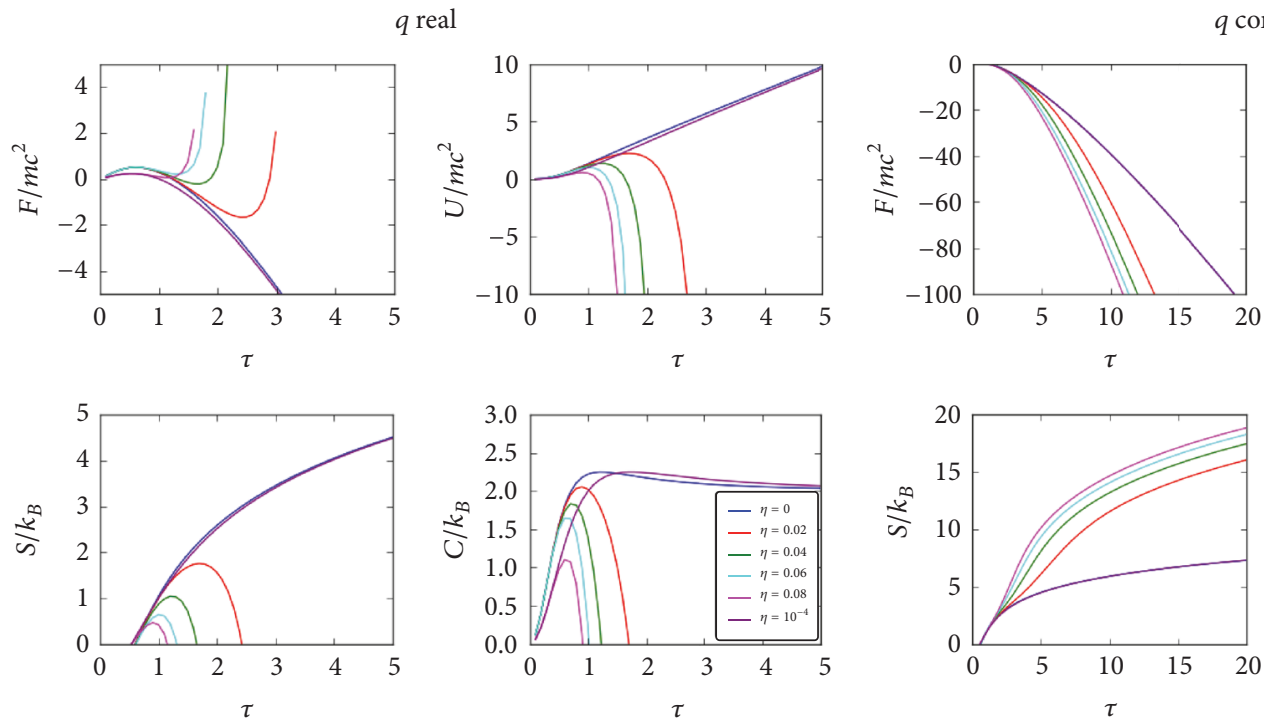

q complex
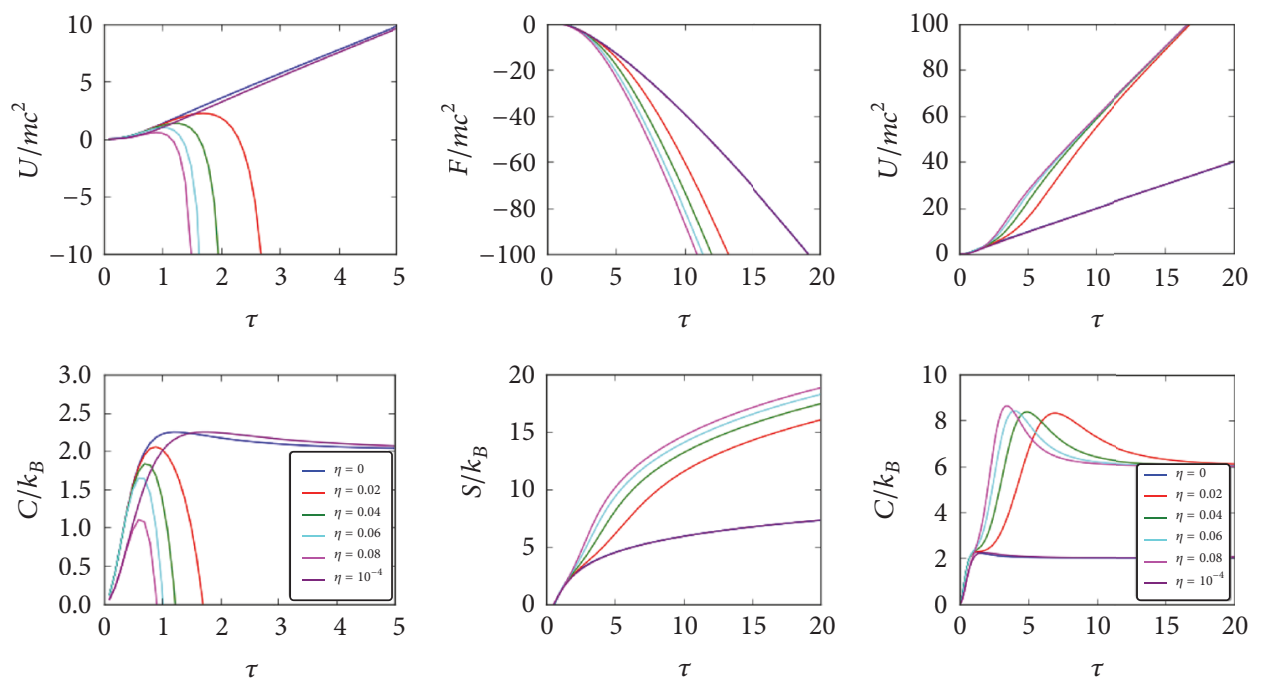

FIGURE 3: Thermal properties of a $q$-deformed Dirac oscillator in one and two dimensions.

structure. The electronic properties of Graphene are exceptionally novel. For instance, the low-energy quasiparticles in Graphene behave as massless chiral Dirac fermions, which has led to the experimental observation of many interesting effects similar to those predicted in the relativistic regime. In the recent study [26], the author has shown, by using an approach based on the effective mass, that the model of a twodimensional DO can be used to describe the thermodynamic properties of Graphene under an uniform magnetic field. By using the formalism of the creation and annihilation operators in complex formalism, he arrives at the following spectrum of energy:

$$
\varsigma_{n}^{ \pm}= \pm \sqrt{2} \frac{\hbar \widetilde{c}}{l_{B}} \sqrt{n},
$$

with $l_{B}=\sqrt{\hbar / e B}$ being the so-called magnetic length and $\widetilde{c}$ the Fermi velocity of electrons in the Graphene. The form of this spectrum of energy is in good agreement with the form of the case of Graphene (see [52]). This form of spectrum of energy in the presence of a deformation $q$ can be written in a pure phase by

$$
\bar{\zeta}_{n}^{ \pm}= \pm \sqrt{2} \frac{\hbar \widetilde{c}}{l_{B}} \sqrt{\frac{\sin (\eta n)}{\sin (\eta)}}
$$

In the approximation of very small deformation, we have

$$
\frac{\zeta_{q n}}{a 1}=\varsigma_{n}^{+}\left(1+\frac{n^{2}}{12} \eta^{2}\right),
$$

with $a 1=\sqrt{2} \hbar \widetilde{c} / l_{B}$, and, consequently, the final partition function of the $q$-deformed version of Graphene is

$$
Z_{q}^{\prime}(\tau, \eta)=Z_{0}^{\prime}+Z_{1}^{\prime},
$$

with

$$
Z_{0}^{\prime}=\tau^{2}+\frac{1}{2}
$$

according to [26], and

$$
Z_{1}^{\prime}=\frac{\eta^{2}}{12 \tau} \sum_{n=0}^{\infty} n^{2} e^{-\sqrt{n} / \tau} .
$$

Here $\tau=k_{B} l_{B} T / \sqrt{2} \hbar \widetilde{c}$. In order to evaluate $Z_{1}$, we use the Euler-Maclaurin formula; so we obtain

$$
Z_{1}^{\prime}=20 \eta^{2} \tau^{5}
$$

Thus, the final form of partition function is

$$
Z_{q}^{\prime}(\tau, \eta)=\tau^{2}+\frac{1}{2}+20 \tau^{5} \eta^{2}
$$

Via (34), the determination of all thermal properties, such as the free energy, the entropy, total energy, and the specific heat, can be obtained through the numerical partition function $Z_{q}^{\prime}(\tau, \eta)$ via the following relations:

$$
\begin{aligned}
& \frac{F}{a 1}=-\frac{1}{\bar{\beta}} \ln (Z)=-\tau \ln (Z), \\
& \frac{U}{a 1}=-\frac{\partial \ln (Z)}{\partial \bar{\beta}}=\tau^{2} \frac{\partial \ln (Z)}{\partial \tau}, \\
& \frac{S}{k_{B}}=\bar{\beta}^{2} \frac{\partial(F / a)}{\partial \bar{\beta}}=\ln (Z)+\tau \frac{\partial \ln (Z)}{\partial \tau}, \\
& \frac{C}{k_{B}}=-\bar{\beta}^{2} \frac{\partial(U / a)}{\partial \bar{\beta}}=2 \tau \frac{\partial \ln (Z)}{\partial \tau}+\tau^{2} \frac{\partial^{2} \ln (Z)}{\partial \tau^{2}} .
\end{aligned}
$$

The thermodynamic quantities are, respectively, plotted in Figure 4. From this figure, we observe that the behavior of the specific heat in the asymptotic regions is greater than that in the case of Graphene: here the limit is $5 k_{B}$. In addition, as in the nondeformed case of Graphene, we can argue this situation by saying that these limits follow the Dulong-Petit law for an ultrarelativistic ideal gas. 


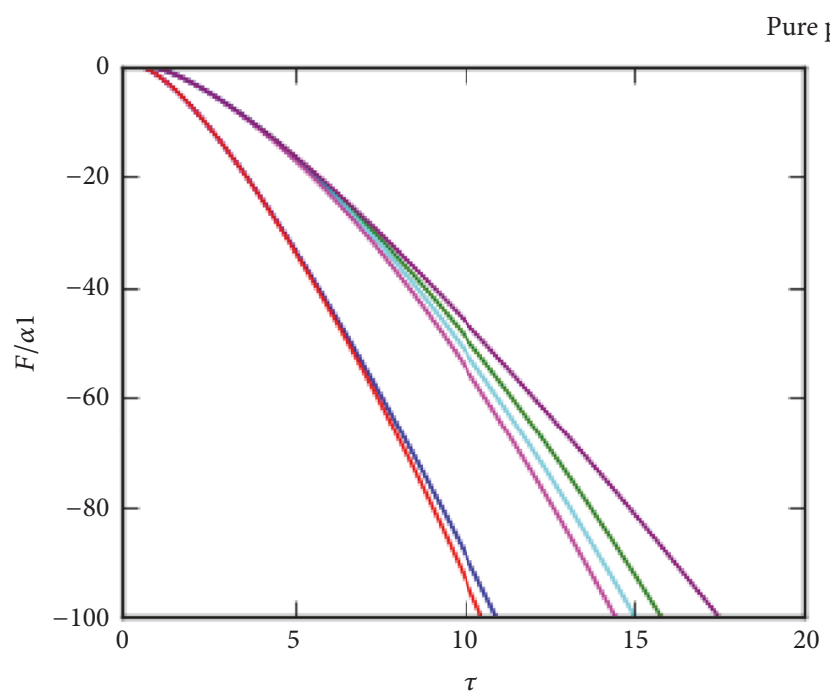

ure phase
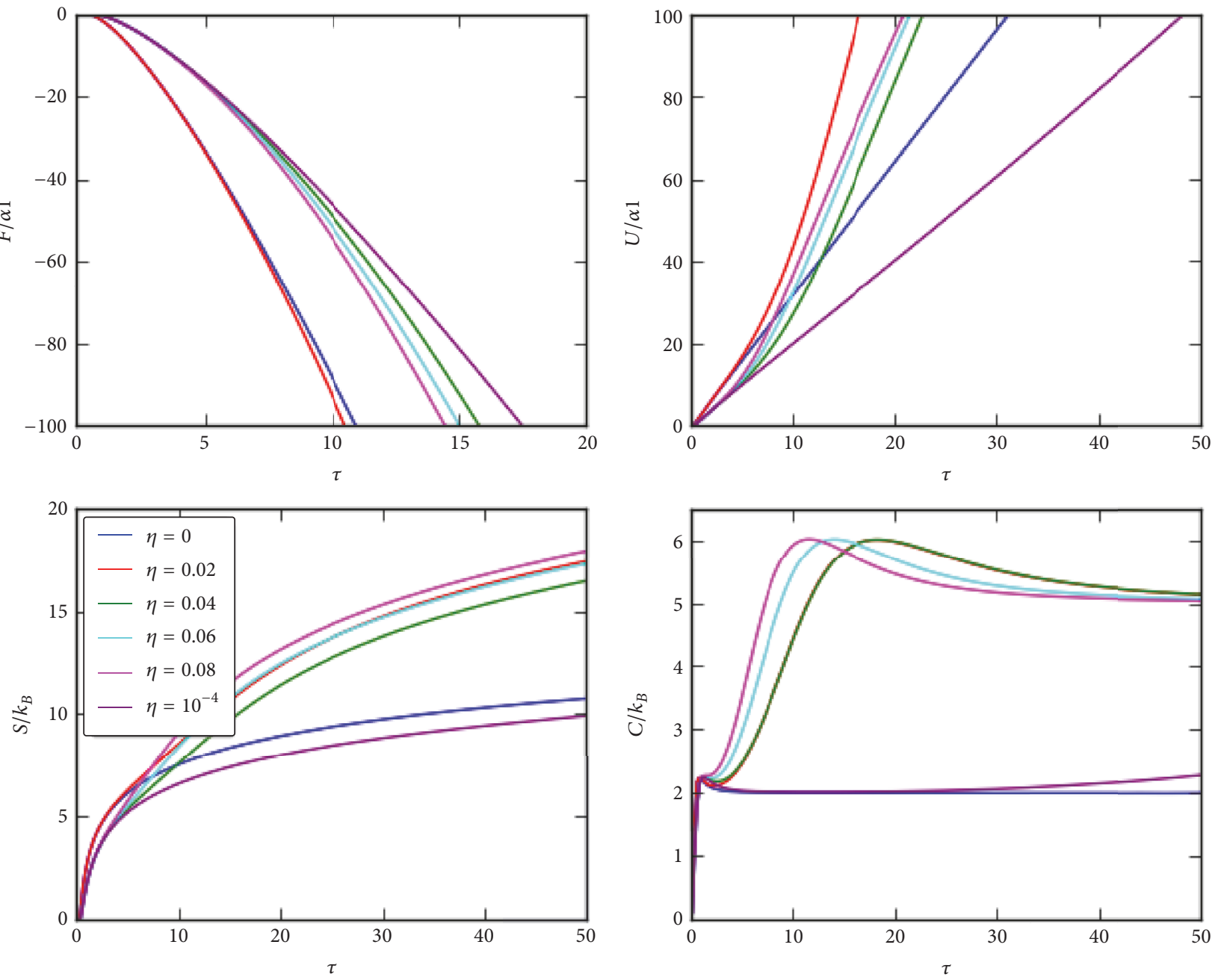

FIGURE 4: Thermal properties of Graphene in its $q$-deformed version.

\section{Conclusion}

In this paper, after a brief preliminary around $q$-deformed oscillator, we studied the DO in this deformation formalism. We have found the eigenvalues and eigenfunctions by introducing $q$-deformed creation and annihilation operators using the complex formalism. It has been shown that eigenvalues of these oscillators can be derived, and especially we have tested them in limited cases where the ordinary results have been well recovered. And treatments of energy eigenvalues for real and complex values of the $q$ parameter were depicted where difference between real and complex cases were shown clearly. It was seen that, for real values of $q$, in the energy eigenvalues we faced rapid rise so that the spectrum expanded. On the contrary, when we set complex values for $q$, we witness that the eigenvalues increased less rapidly than real case.

The connection between our $q$-deformed DO with quantum optics is well established via JC and AJC models, and the existence of well-known $q$-deformed version of Zitterbewegung in relativistic quantum dynamics has been discussed. In the case of very small deformation, we have calculated in the pure phase case $\left(q=e^{i \eta}\right)$ the partition function and all thermal quantities such as the free energy, total energy, entropy, and specific heat. As an application, we have extended our results to the case of Graphene.

\section{Conflicts of Interest}

The authors declare that they have no conflicts of interest.

\section{References}

[1] D. Bonatsost, L. Britot, D. Menezes, and J. Phys, "Thermal properties of graphene in its q-deformed version," Journal of Physics A: Mathematical and General, vol. 26, pp. 895-904, 1993.

[2] L. C. Biedenharn, "The quantum group $\mathrm{SU}_{q}(2)$ and a $q$-analogue of the boson operators," Journal of Physics. A: Mathematical and General, vol. 22, no. 18, pp. L873-L878, 1989.

[3] A. J. Macfarlane, "On $q$-analogues of the quantum harmonic oscillator and the quantum group SU(2)," Journal of Physics. A. Mathematical and General, vol. 22, no. 21, pp. 4581-4588, 1989. 
[4] P. P. Kulish and E. V. Damaskinsky, "On the $q$ oscillator and the quantum algebra $\mathrm{su}_{q}(1,1)$," Journal of Physics. A. Mathematical and General, vol. 23, no. 9, pp. L415-L419, 1990.

[5] Y. J. Ng, "Comment on the q-analogues of the harmonic oscillator," Journal of Physics. A. Mathematical and General, vol. 23, no. 6, pp. 1023-1027, 1990.

[6] H. Ui and N. Aizawa, "-analogue of boson commutator and the quantum groups $\mathrm{SU}_{q}(2)$ and $\mathrm{SU}_{q}(1,1)$," Modern Physics Letters $A$, vol. 5, no. 4, pp. 237-242, 1990.

[7] A. Lorek, A. Ruffing, and J. Wess, "A q-deformation of the harmonic oscillator," Zeitschrift für Physik, vol. 74, no. 2, pp. 369-377, 1997.

[8] I. L. Cooper and R. K. Gupta, " $q$-deformed Morse oscillator," Physical Review. A. Third Series, vol. 52, no. 2, pp. 941-948, 1995.

[9] A. Lavagno, A. M. Scarfone, and P. N. Swamy, "Classical and quantum $q$-deformed physical systems," The European Physical Journal C. Particles and Fields, vol. 47, no. 1, pp. 253-261, 2006.

[10] J. Črnugelj, M. Martinis, and V. Mikuta-Martinis, "JaynesCummings model and the deformed-oscillator algebra," Physics Letters. A, vol. 188, no. 4-6, pp. 347-354, 1994.

[11] A. M. Gavrilik, I. I. Kachurik, and A. V. Lukash, "New version of q-deformed supersymmetric quantum mechanics," Ukrainian Journal of Physics, vol. 58, no. 11, pp. 1025-1032, 2013.

[12] M. S. Abdalla, H. Eleuch, and J. Appl, "Exact analytic solutions of the Schrödinger equations for some modified $q$-deformed potentials," Journal of Applied Physics, vol. 115, Article ID 234906, 2014.

[13] S. Cai, G. Su, and J. Chen, "Thermostatistic properties of a q-deformed ideal Fermi gas with a general energy spectrum," Journal of Physics. A. Mathematical and Theoretical, vol. 40, no. 37, pp. 11245-11254, 2007.

[14] W. S. Chung, " $q$-deformed tamm-dancoff oscillators: representation, fermionic extension and physical application," International Journal of Modern Physics B, vol. 29, Article ID 1550177, 2015.

[15] W. S. Chung, "New q-deformed fermionic oscillator algebra and thermodynamics," Journal of Advanced Physics, vol. 4, pp. 1-4, 2015.

[16] A. Algin and M. Senay, "Fermionic image-deformation and its connection to thermal effective mass of a quasiparticle," Physica A, vol. 447, pp. 232-246, 2016.

[17] D. Itô, K. Mori, and E. Carriere, "An example of dynamical systems with linear trajectory," Nuovo Cimento A, vol. 51, p. 1119, 1967.

[18] M. Moshinsky and A. Szczepaniak, "The Dirac oscillator," Journal of Physics. A. Mathematical and General, vol. 22, no. 17, pp. L817-L819, 1989.

[19] R. P. Martinez-y-Romero, A. L. Salas-Brito, and J. Math, "Conformal invariance in a Dirac oscillator," Journal of Mathematical Physics, vol. 33, p. 1831, 1992.

[20] M. Moreno and A. Zentella, "Covariance, CPT and the FoldyWouthuysen transformation for the Dirac oscillator," Journal of Physics. A. Mathematical and General, vol. 22, no. 17, pp. L821L825, 1989.

[21] J. Benitez, R. P. Martinez y Romero, H. N. Nunez-Yepez, and A. L. Salas-Brito, "Solution and hidden supersymmetry of a Dirac oscillator," Physical Review Letters, vol. 64, no. 14, pp. 1643-1645, 1990.

[22] C. Quesne, V. M. Tkachuk, J. Phys, and A. Math, "Dirac oscillator with nonzero minimal uncertainty in position," Journal of Physics A: Mathematical and General, vol. 41, pp. 1747-1765, 2005.
[23] A. Boumali and H. Hassanabadi, "The thermal properties of a two-dimensional Dirac oscillator under an external magnetic field," The European Physical Journal Plus, vol. 128, p. 124, 2013.

[24] A. Boumali and H. Hassanabadi, "Exact solutions of the $(2+1)$ dimensional Dirac oscillator under a magnetic field in the presence of a minimal length in the noncommutative phasespace," Zeitschrift für Naturforschung A, vol. 70, pp. 619-627, 2015.

[25] A. Boumali, "The one-dimensional thermal properties for the relativistic harmonic oscillators," Electronic Journal of Theoretical Physics, vol. 12, no. 32, pp. 1-10, 2015.

[26] A. Boumali, "Thermodynamic properties of the graphene in a magnetic field via the two-dimensional Dirac oscillator," Physica Scripta, vol. 90, Article ID 045702, 2015.

[27] P. Strange and L. H. Ryder, "The Dirac oscillator in a rotating frame of reference," Physics Letters. A, vol. 380, no. 42, pp. 3465$3468,2016$.

[28] J. A. Franco-Villafañe, E. Sadurní, S. Barkhofen, U. Kuhl, F. Mortessagne, and T. H. Seligman, "First Experimental Realization of the Dirac Oscillator," Physical Review Letters, vol. 111, Article ID 170405, 2013.

[29] E. T. Jaynes and F. W. Cummings, "Comparison of Quantum and Semiclassical Radiation Theories with Application to the Beam Maser," Proceedings of the IEEE, vol. 51, no. 1, pp. 89-109, 1963.

[30] F. W. Cummings, "Stimulated emission of radiation in a single mode," Physical Review, vol. 140, no. 4A, pp. A1051-A1056, 1965.

[31] A. Bermudez, M. A. Martin-Delgado, and E. Solano, "Exact mapping of the $2+1$ Dirac oscillator onto the Jaynes-Cummings model: Ion-trap experimental proposal," Physical Review A, vol. 76, Article ID 041801, 2007.

[32] I. Lutzenko, V. Spiridonov, and A. Zhedanov, "On the spectrum of a q-oscillator with a linear interaction," Physics Letters. A, vol. 204, no. 3-4, pp. 236-242, 1995.

[33] L. M. Kuang, "A $q$-deformed harmonic oscillator in a finitedimensional Hilbert space," Journal of Physics. A. Mathematical and General, vol. 26, no. 20, pp. L1079-L1083, 1993.

[34] A. S. Zhedanov, "Non-classical q-oscillator realization of the quantum SU(2) algebra," Journal of Physics. A. Mathematical and General, vol. 25, no. 12, pp. L713-L717, 1992.

[35] M. Chaichian, "Quantum algebra as the dynamical symmetry of the deformed Jaynes-Cummings model," Physical Review Letters, vol. 65, p. 980, 1990.

[36] M. L. Strekalov, "An accurate closed-form expression for the partition function of Morse oscillators," Chemical Physics Letters, vol. 439, pp. 209-212, 2007.

[37] C. Jia, L. Zhang, and C. Wang, "Thermodynamic properties for the lithium dimer," Chemical Physics Letters, vol. 667, pp. 211215, 2017.

[38] X. Songa, C. Wanga, and C. Jia, "Thermodynamic properties for the sodium dimer," Chemical Physics Letters, vol. 673, pp. 50-55, 2017.

[39] C. Jiaa, C. Wanga, L. Zhanga, X. Penga, R. Zenga, and X. Youa, "Partition function of improved Tietz oscillators," Chemical Physics Letters, vol. 676, pp. 150-153, 2017.

[40] S. A. Alavi and S. Rouhani, "Exact analytical expression for magnetoresistance using quantum groups," Physics Letters. A, vol. 320, no. 4, pp. 327-332, 2004.

[41] M. R-Monteiro, L. M. C. S. Rodrigues, and S. Wulck, "Quantum algebraic nature of the phonon spectrum in $4 \mathrm{He}$," Physical Review Letters, vol. 76, no. 7, 1996. 
[42] R. S. Johal and R. K. Gupta, “Two Parameter Quantum Deformation of $U(2) \supset U(1)$ Dynamical Symmetry and the Vibrational Spectra of Diatomic Molecules," International Journal of Modern Physics. E. Nuclear Physics, vol. 7, no. 5, pp. 553-557, 1998.

[43] Z. Chang and H. Yan, "Diatomic-molecular spectrum in view of quantum group theory," Physical Review A, vol. 44, no. 11, pp. 7405-7413, 1991.

[44] D. Bonatsos, C. Daskaloyannis, and P. Kolokotronis, "Coupled Q-oscillators as a model for vibrations of polyatomic molecules," Journal of Chemical Physics, vol. 106, p. 605, 1997.

[45] M. Xie, X.-W. Hou, and Z.-Q. Ma, " $q$-Deformed harmonic oscillators applied to the vibrational spectrum of methane," Chemical Physics Letters, vol. 262, no. 1-2, pp. 1-7, 1996.

[46] M. Xie, X.-W. Hou, and Z.-Q. Ma, Physics Letters, vol. 667, pp. 211-215, 2017.

[47] P. K. Bera and J. Datta, "Spectral inverse problem for $q$ deformed harmonic oscillator," Pramana, vol. 67, no. 6, pp. 1023-1035, 2006.

[48] M. Torres, E. Sadurní, and T. H. Seligman, "The DiracMoshinsky oscillator coupled to an external field and its connection to quantum optics," in Proceedings of the AIP Conference Proceedings, vol. 1323, pp. 301-312, 2010.

[49] N. Hatami and M. R. Setare, "The $q$-deformed Dirac oscillator in $2+1$ dimensions," Physics Letters. A, vol. 380, no. 42, pp. 34693472, 2016.

[50] P. Neskovic and B. V. Urosevic, "Quantum oscillators: applications in statistical mechanics," International Journal of Modern Physics A, vol. 7, no. 14, pp. 3379-3388, 1992.

[51] H. Liu, F. Men, Y. Li, B. Guan, and M. Zhang, “Thermostatistic properties of the relativistic $q$-deformed ideal Fermi gas at high temperature," Wuhan University Journal of Natural Sciences, vol. 15, no. 1, pp. 57-63, 2010.

[52] Z. Jiang, E. A. Henriksen, L. C. Tung et al., "Infrared spectroscopy of Landau levels in graphene," Physical Review Letters, vol. 98, Article ID 197403, 2007. 

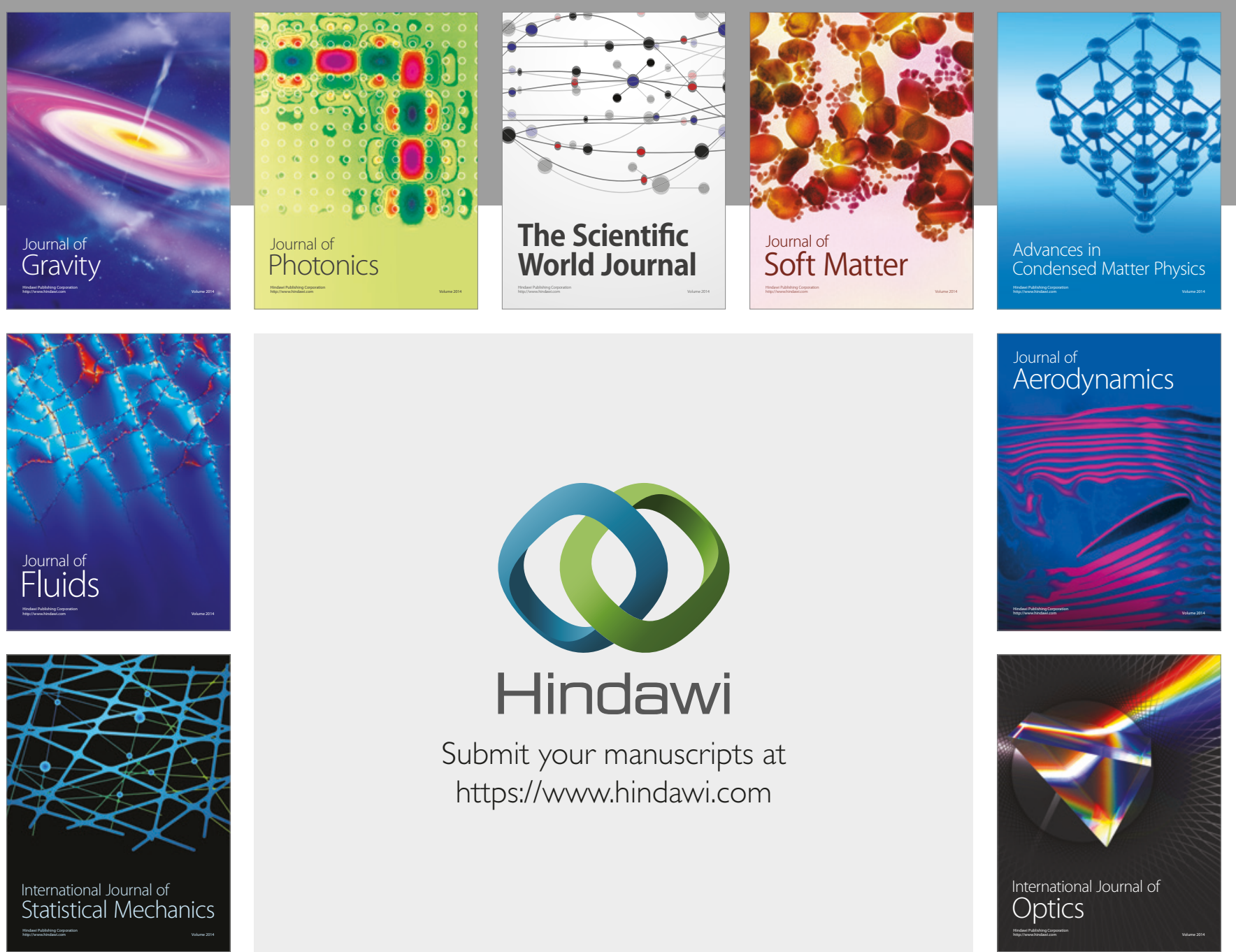

Submit your manuscripts at

https://www.hindawi.com
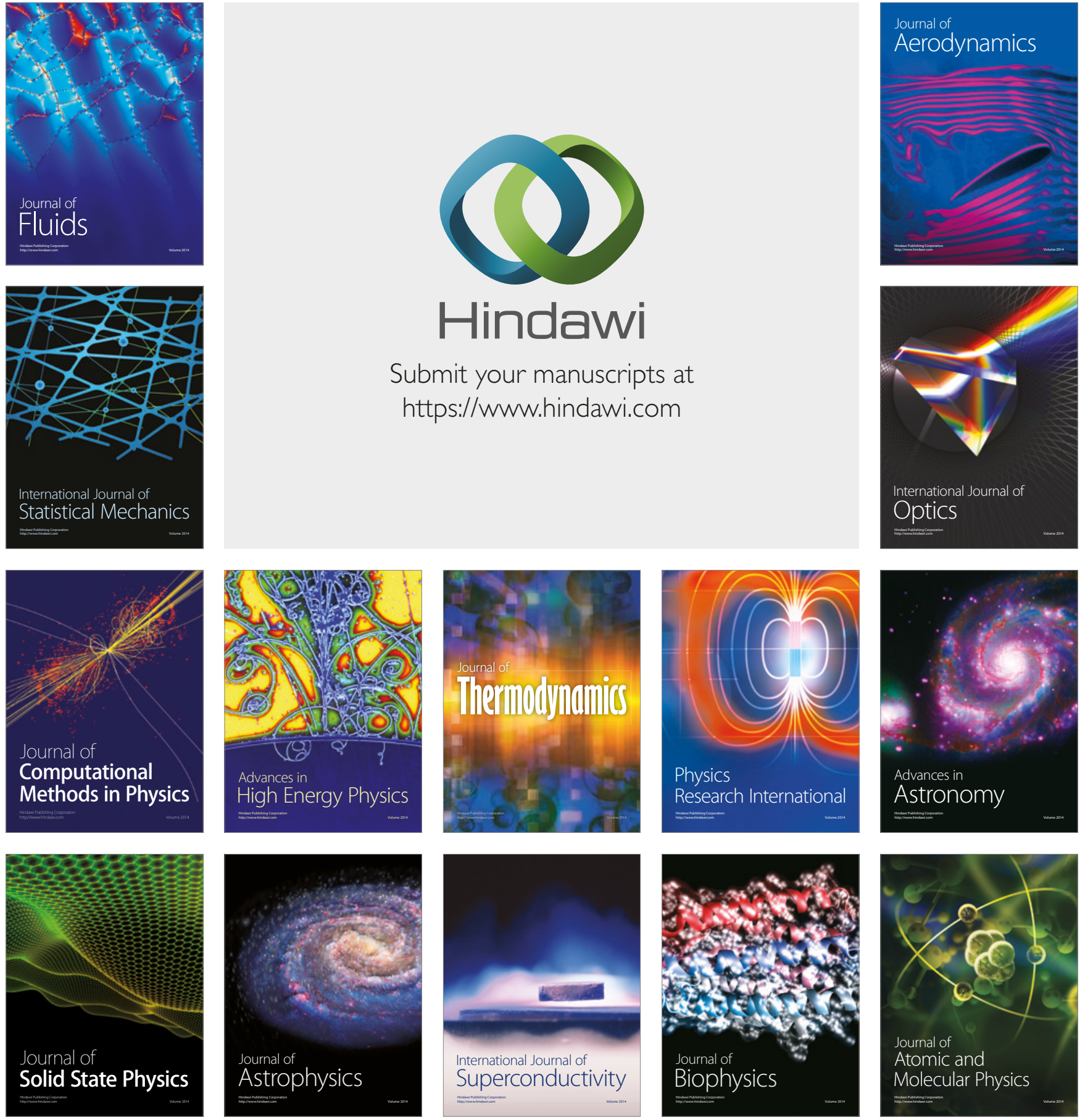